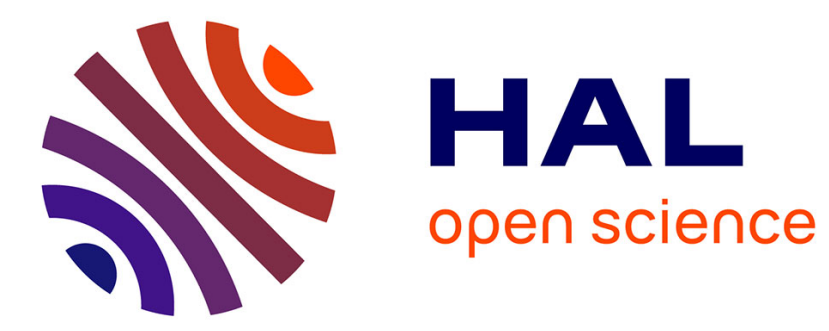

\title{
Quasiparticle and optical properties of BeH2
}

S. Lebegue, C. M. Araujo, O. Eriksson, B. Arnaud, M. Alouani, R. Ahuja

\section{To cite this version:}

S. Lebegue, C. M. Araujo, O. Eriksson, B. Arnaud, M. Alouani, et al.. Quasiparticle and optical properties of BeH2. Journal of Physics: Condensed Matter, 2007, 19, pp.036223. 10.1088/09538984/19/3/036223. hal-00212165

\section{HAL Id: hal-00212165 \\ https://hal.science/hal-00212165}

Submitted on 2 Mar 2020

HAL is a multi-disciplinary open access archive for the deposit and dissemination of scientific research documents, whether they are published or not. The documents may come from teaching and research institutions in France or abroad, or from public or private research centers.
L'archive ouverte pluridisciplinaire HAL, est destinée au dépôt et à la diffusion de documents scientifiques de niveau recherche, publiés ou non, émanant des établissements d'enseignement et de recherche français ou étrangers, des laboratoires publics ou privés.

\section{(c)(1)}

Distributed under a Creative Commons Attribution| 4.0 International License 


\title{
Quasiparticle and optical properties of $\mathrm{BeH}_{2}$
}

\author{
S Lebègue ${ }^{1,2}$, C Moysés Araújo ${ }^{1}$, O Eriksson $^{1}$, B Arnaud $^{3}$, M Alouani $^{4}$ \\ and R Ahuja ${ }^{1}$ \\ ${ }^{1}$ Department of Physics, Uppsala University, SE-75121 Uppsala, Sweden \\ ${ }^{2}$ Laboratoire de Cristallographie et de Modélisation des Matériaux Minéraux et Biologiques, \\ UMR 7036, CNRS-Université Henri Poincaré, BP 239, F-54506 Vandoeuvre-lès-Nancy, France \\ ${ }^{3}$ Groupe Matière condensée et Matériaux (GMCM), Campus de Beaulieu, Batiment 11A, 35042 \\ Rennes cedex, France \\ ${ }^{4}$ Institut de Physique et de Chimie des Matériaux de Strasbourg, UMR 7504 CNRS-ULP, 23 rue \\ du Loess, 67034 Strasbourg, France
}

\begin{abstract}
The quasiparticle and optical properties of $\mathrm{BeH}_{2}$ are computed by means of the all-electron GW approximation in conjunction with the projector augmented wave (PAW) method. The GW approximation, through the calculation of the self-energy and the optical dielectric function in the random phase approximation, shows that $\mathrm{BeH}_{2}$ is a large band gap insulator. The results are discussed in view of future experiments.
\end{abstract}

\section{Introduction}

The search for new materials with suitable properties in view of hydrogen storage is a continuous challenge, bridging the gap between fundamental science and applied physics. Some of the most promising candidates are the light metal hydrides, like $\mathrm{MgH}_{2}, \mathrm{NaAlH}_{4}$, and $\mathrm{LiBH}_{4}$, among many others [1, 2]. Several key features to obtain usable hydrogen-based fuel cells are already known. For example, the need for an efficient absorption/desorption dynamic of hydrogen is clear, as is the need for the materials used in applications to be inexpensive. A material which has recently drawn attention along these lines is beryllium dihydride $\left(\mathrm{BeH}_{2}\right)$. Although this compound is difficult to prepare in pure crystalline form, it presents many interesting features in view of technological applications. $\mathrm{BeH}_{2}$ is of interest for hydrogen storage due to its low mass and high hydrogen content. However, its toxicity limits its use enormously at present, except maybe as a rocket fuel in outer space. Also, varying the amount of hydrogen in $\mathrm{BeH}_{2}$ together with alloying can possibly lead to properties relevant for switchable mirror technology, like for the parent compound $\mathrm{MgH}_{2}$ [3, 4]. For all these reasons, there has recently been a renewal of interest in beryllium hydride in the scientific community [2, 5-8]. In particular, several authors [9, 10] have predicted the groundstate properties of $\mathrm{BeH}_{2}$ using density functional theory (DFT). Also, these authors have been 
able to reproduce from an ab initio calculation the correct experimental structure [11] and to predict a series of structural modifications under pressure, but we are not aware of any study of excited states. Since experiments like photoemission, inverse photoemission, as well as optical spectroscopy, are usual tools to probe the electronic structure of materials, we decided to conduct an investigation of the excited-state properties of $\mathrm{BeH}_{2}$.

Our paper is organized as follows. In the next section, we briefly review the methods used in this work, namely the GW approximation (GWA) to the self-energy and the dipolar approximation to the dielectric constant. Then, we present the quasiparticle band structure as well as the dielectric function of $\mathrm{BeH}_{2}$; the self-energy correction and the optical spectra are discussed. Finally we summarize and offer our conclusions.

\section{Formalism}

The purpose of the GW approximation $[12,13]$ is to overcome the difficulty of the DFT in the calculation of the excited state properties. Within this formalism, the usual Kohn-Sham equations are replaced by quasiparticle (QP) equations written as (the QP energies and QP wavefunctions being denoted respectively $E_{n}(\mathbf{k})$ and $\left.\psi_{\mathbf{k} n}(\mathbf{r})\right)$

$$
\left(T+V_{\mathrm{ext}}+V_{\mathrm{h}}\right) \psi_{\mathbf{k} n}(\mathbf{r})+\int \mathrm{d}^{3} r^{\prime} \Sigma\left(\mathbf{r}, \mathbf{r}^{\prime}, E_{n}(\mathbf{k})\right) \psi_{\mathbf{k} n}\left(\mathbf{r}^{\prime}\right)=E_{n}(\mathbf{k}) \psi_{\mathbf{k} n}(\mathbf{r}),
$$

where $T, V_{\text {ext }}, V_{\mathrm{h}}$, and $\Sigma$ are, respectively, the kinetic energy operator, the external potential due to the ion cores, the average electrostatic (Hartree) potential, and the electron self-energy operator. This last quantity is written as the product of the one-electron Green's function $G$ and the screened Coulomb interaction $W[12,13]$ :

$$
\Sigma\left(\mathbf{r}, \mathbf{r}^{\prime}, \omega\right)=\frac{\mathrm{i}}{2 \pi} \int \mathrm{d} \omega^{\prime} G\left(\mathbf{r}, \mathbf{r}^{\prime}, \omega+\omega^{\prime}\right) \mathrm{e}^{\mathrm{i} \delta \omega^{\prime}} W\left(\mathbf{r}, \mathbf{r}^{\prime}, \omega^{\prime}\right),
$$

where $\delta$ is a positive infinitesimal. The quasiparticle energies $E_{n}(\mathbf{k})$ are then obtained by a first-order expansion of the self-energy:

$\operatorname{Re} E_{n}(\mathbf{k})=\epsilon_{n}(\mathbf{k})+Z_{n \mathbf{k}} \times\left[\left\langle\Psi_{\mathbf{k} n}\left|\operatorname{Re} \Sigma\left(\mathbf{r}, \mathbf{r}^{\prime}, \epsilon_{n}(\mathbf{k})\right)\right| \Psi_{\mathbf{k} n}\right\rangle-\left\langle\Psi_{\mathbf{k} n}\left|V_{\mathrm{xc}}^{\mathrm{DFT}}(r)\right| \Psi_{\mathbf{k} n}\right\rangle\right]$

and the renormalization factor $Z_{n \mathbf{k}}$ corresponds to

$$
Z_{n \mathbf{k}}=\left[1-\left\langle\Psi_{\mathbf{k} n}\left|\frac{\partial}{\partial \omega} \operatorname{Re} \Sigma\left(\mathbf{r}, \mathbf{r}^{\prime}, \omega=\epsilon_{n}(\mathbf{k})\right)\right| \Psi_{\mathbf{k} n}\right\rangle\right]^{-1}
$$

Since the evaluation of the screened Coulomb interaction $W$ is computationally heavy, we have not used the random-phase approximation (RPA) as implemented in [14] but rather the plasmon-pole (PIP) approximation, which avoids the calculation of the full dielectric function as implemented in [15]. Therefore the imaginary part of the self-energy is not accessible for the present work, but since we are interested mainly in the quasiparticle band structure and in the optical properties, this is not an important limitation. Apart from a slight overestimation of the interband transitions (see [14]) the produced quasiparticle energies are in good agreement with the full GW-RPA for 'sp' materials. Then, we use the calculated QP eigenvalues to compute the optical properties. The macroscopic dielectric function (when ignoring local field effects) is written as

$$
\epsilon(\omega)=\epsilon_{1}(\omega)+\mathrm{i} \epsilon_{2}(\omega)=\lim _{\mathbf{q} \rightarrow 0} \epsilon_{0,0}(\mathbf{q}, \omega)
$$

Following the general formulation of Adler and Wiser [16-18], the dielectric function $\epsilon_{\mathbf{G}, \mathbf{G}^{\prime}}(\mathbf{q}, \omega)$ in the RPA is given by

$\epsilon_{\mathbf{G}, \mathbf{G}^{\prime}}(\mathbf{q}, \omega)=\delta_{\mathbf{G}, \mathbf{G}^{\prime}}-\frac{8 \pi}{\Omega\left|\mathbf{q}+\mathbf{G} \| \mathbf{q}+\mathbf{G}^{\prime}\right|}{ }_{l, m, \mathbf{k}} \frac{\left[n_{l \mathbf{k}-\mathbf{q}}-n_{m \mathbf{k}}\right] M_{\mathbf{G}}^{l m}(\mathbf{k}, \mathbf{q}) M_{\mathbf{G}^{\prime}}^{l m}(\mathbf{k}, \mathbf{q})^{*}}{\epsilon_{l \mathbf{k}-\mathbf{q}}^{q p}-\epsilon_{m \mathbf{k}}^{q p}+\omega+\mathrm{i} \delta}$ 


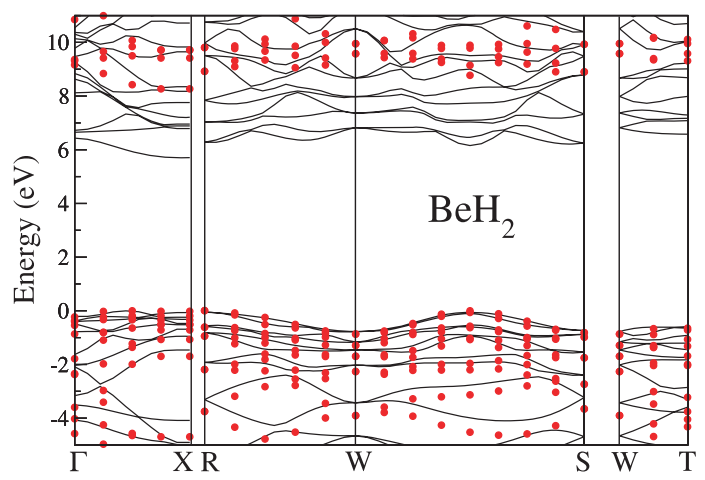

Figure 1. GGA band structure (full lines) and quasiparticle corrections (red dots) for $\mathrm{BeH}_{2}$. The top of the valence state is at the zero of the energy scale.

where $\delta$ is a positive infinitesimal, $n_{m \mathbf{k}}$ and $\epsilon_{m \mathbf{k}}^{q p}$ being respectively the occupation number and the one-particle energy for a state specified by the band index $m$ and the wavevector $\mathbf{k}$. The matrix elements $M_{\mathbf{G}}^{l m}(\mathbf{k}, \mathbf{q})$ are written as

$$
M_{\mathbf{G}}^{l m}(\mathbf{k}, \mathbf{q})=\left\langle\Psi_{\mathbf{k}-\mathbf{q} l}\left|\mathrm{e}^{-\mathrm{i}(\mathbf{q}+\mathbf{G}) \mathbf{r}}\right| \Psi_{\mathbf{k} m}\right\rangle .
$$

In the numerical procedure, we first calculate the imaginary part of the dielectric function $\epsilon_{2}(\omega)$. The Brillouin zone (BZ) integration is conducted by using the tetrahedron method [19, 20] as described in [21]. Finally, the real part of $\epsilon(\omega)$ is obtained by using a Kramers-Kronig transformation.

This method has been used successfully for a wide range of materials [22-24] in the past. In particular, our implementation has been successfully applied to a number of important materials for hydrogen storage like $\mathrm{LiH}$ [25] and $\mathrm{MgH}_{2}$ [26]. It is therefore quite reliable, and in the next section we present our results concerning $\mathrm{BeH}_{2}$.

\section{Results and discussion}

As presented in the previous section, the present GW implementation is a perturbative theory (see equation (3)). We have therefore to conduct a standard DFT calculation to be used as a basis for the GW calculation. For this purpose, we used the projector augmented waves method (PAW) [27] to calculate the ground state of $\mathrm{BeH}_{2}$. In the low-pressure phase, this compound crystallizes in the Ibam space group (number 72) with 18 atoms per primitive cell. For all our calculations, we used the experimental lattice parameters [11] and the generalized gradient approximation (GGA) of Perdew et al [28] for the exchange-correlation potential. This part of the calculation is very similar to the one already performed elsewhere $[9,10]$ and will not be presented in this paper. We prefer therefore to concentrate on the calculation of the excited-state properties.

In figure 1 we present the GGA (full lines) and GW (dots) band structures for $\mathrm{BeH}_{2}$ along some high-symmetry directions of the BZ. All the convergence parameters of our calculation have been carefully optimized to produce reliable results: we used a $k$-point mesh of $3 \times 3 \times 3$, the size of the matrix $W$ representing the screened interaction is $249 \times 249$, and 100 bands were used for the summation over conduction bands.

It is found that $\mathrm{BeH}_{2}$ is a direct band gap insulator at the high-symmetry point $\mathrm{X}$, the value of the band gap being $5.78 \mathrm{eV}$ within the GGA and $8.27 \mathrm{eV}$ within the $\mathrm{GW}$ approximation. Our GGA result is in good agreement with the value of $5.51 \mathrm{eV}$ reported in [10]. In fact, 


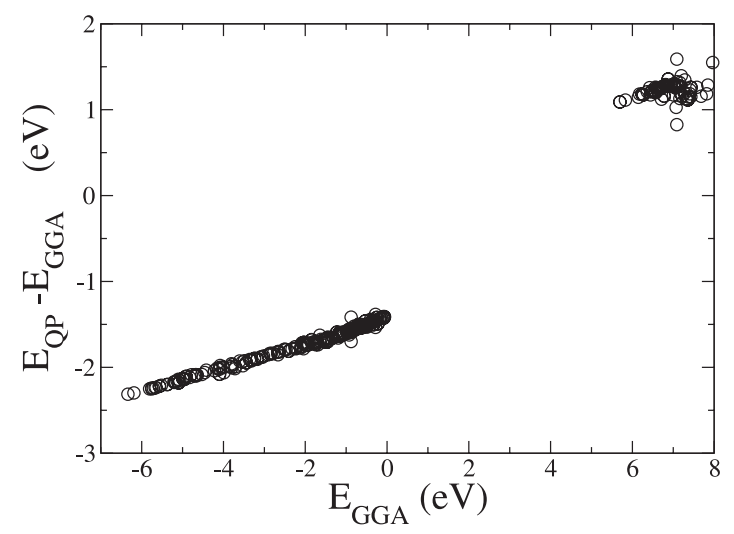

Figure 2. Quasiparticle correction $E_{\mathrm{QP}}-E_{\mathrm{GGA}}$ as a function of $E_{\mathrm{GGA}}$ for $\mathrm{BeH}_{2}$.

Table 1. Quasiparticle energies of interband transitions in $\mathrm{eV}$ at the high-symmetry points $\Gamma, \mathrm{X}, \mathrm{R}$, $\mathrm{W}, \mathrm{S}$, and $\mathrm{T}$ for $\mathrm{BeH}_{2}$. The value corresponding to the minimum gap is underlined.

\begin{tabular}{llc}
\hline & GGA $(\mathrm{eV})$ & $\mathrm{GW}(\mathrm{eV})$ \\
\hline $\boldsymbol{\Gamma}_{v} \rightarrow \boldsymbol{\Gamma}_{c}$ & 6.64 & 9.37 \\
$\mathbf{X}_{v} \rightarrow \mathbf{X}_{c}$ & $\underline{5.78}$ & $\underline{8.27}$ \\
$\mathbf{R}_{v} \rightarrow \mathbf{R}_{c}$ & 6.35 & 8.92 \\
$\mathbf{W}_{v} \rightarrow \mathbf{W}_{c}$ & 7.57 & 10.49 \\
$\mathbf{S}_{v} \rightarrow \mathbf{S}_{c}$ & 7.00 & 9.71 \\
$\mathbf{T}_{v} \rightarrow \mathbf{T}_{c}$ & 7.15 & 9.96 \\
\hline
\end{tabular}

the eigenvalues of the last valence band at several high-symmetry points $(\mathrm{X}, \mathrm{R}$, and some points along the $\Gamma \rightarrow \mathrm{X}$ and $\mathrm{W} \rightarrow \mathrm{S}$ directions) are almost degenerate, making it difficult to determine the exact nature of the band gap. Even if the band gap is in fact indirect, the difference in energy from that of the smallest direct band gap would be so small that it will make no difference in view of applications. In table 1, we provide the values of the transition energies for a set of high-symmetry points, which is useful for a detailed comparison with future photoemission experiments. As for the energy band gap, the values of the transitions are all enhanced when using the GW approximation instead of the GGA.

Contrary to common assumptions, the GW correction is not always strictly equivalent to a rigid shift [29]. This is illustrated for the present case in figure 2, where the quasiparticle correction $E_{\mathrm{QP}}-E_{\mathrm{GGA}}$ is shown as a function of $E_{\mathrm{GGA}}$.

For the valence band (state below $0 \mathrm{eV}$ for $E_{\mathrm{GGA}}$ ), it is seen clearly that the correction is more important for the states deep in energy than for the top of the valence band, but the overall correction can be easily fitted by a linear function. However, for the conduction band the behaviour seems to be highly irregular. Some points clearly cannot be fitted by a linear function; this can be related to different localization of the electron as a function of the $k$ points in the BZ (see [29] for a discussion of the GW correction for different kinds of states). Also, notice that the bandwidth is increased when using GW in comparison with GGA, which is an effect observed previously [30].

In this paragraph we focus on the optical properties of $\mathrm{BeH}_{2}$. Since optical spectroscopy is an important probe for studying the electronic structure of materials, precise $a b$ initio calculations are therefore needed to compute optical spectra to be able to compare the results to experiment. We have consequently used the formalism presented above to calculate the dielectric function of $\mathrm{BeH}_{2}$, as presented in figures 3 and 4. 


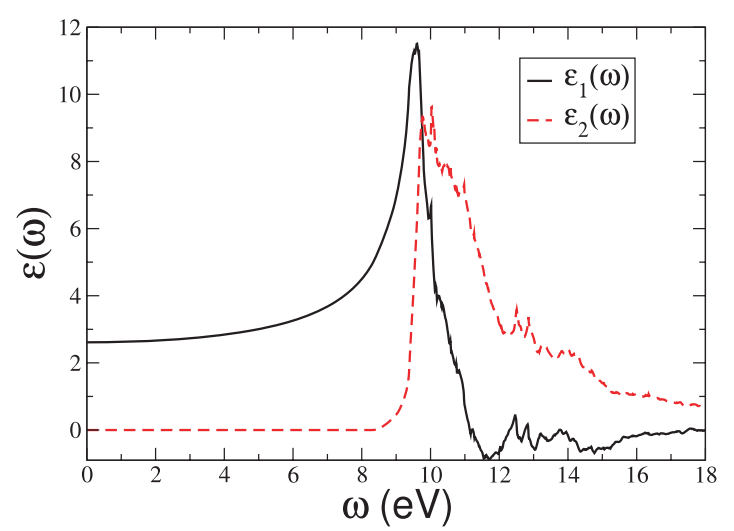

Figure 3. Real and imaginary dielectric functions for $\mathrm{BeH}_{2}$ averaged over the three nonequivalent directions.

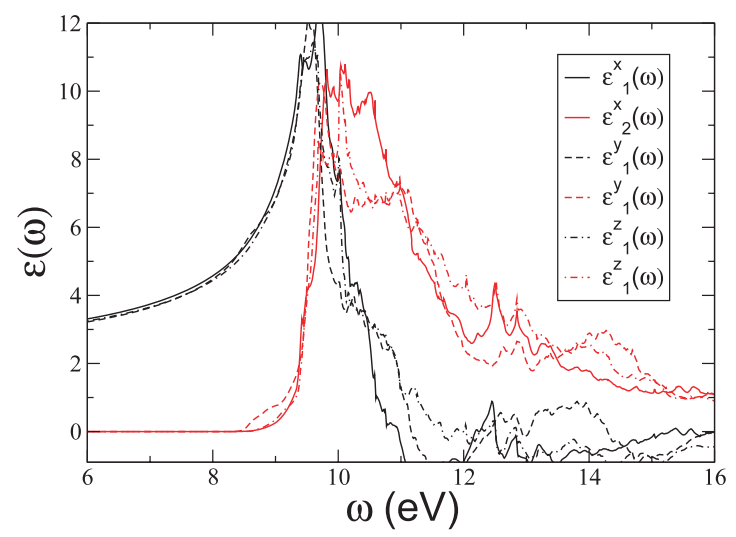

Figure 4. Real and imaginary dielectric functions for $\mathrm{BeH}_{2}$ for each direction $(x, y$, and $z)$.

The three dielectric functions (one for each nonequivalent direction), as presented in figure 4, do not show any distinguishable features; therefore we will only comment on the averaged dielectric function (figure 3). To avoid the heavy computation of GW quasiparticle energies for a large number of $k$ points and bands, we mimic the self-energy effect by a scissor shift of $2.49 \mathrm{eV}$, which corresponds to the correction at the $\mathrm{X}$ point. The shape of the imaginary part is characterized by a sharp increase around $9 \mathrm{eV}$, corresponding to a sudden increase of the number of available transitions around this energy, as can be seen from the band structure. The dielectric function shows some peaks, but these are difficult to correlate with the band structure due to the high number of bands lying in the same range of energy. Also the static dielectric constants are found to be 3.1 without the quasiparticle corrections (not shown here) for the eigenvalues, and 2.6 when using the QP shift. This classifies $\mathrm{BeH}_{2}$ as a large band gap insulator, as already seen from the band structure. However to get a meaningful value, one should either include local field effects (related to non-diagonal elements of the dielectric matrix and ignored here) in the DFT calculation or include both excitonic (the electron-hole interaction that occurs when promoting the excited electrons from the valence band to the conduction band) and local field effects together with the self-energy correction. This was not attempted here due to the heavy computational task that it would represent. 


\section{Conclusion}

We have presented a study of the quasiparticle and optical properties of $\mathrm{BeH}_{2}$. It is found that the band gap is predicted to have a value of approximately $8.3 \mathrm{eV}$, and that it occurs at the high-symmetry point $\mathrm{X}$. Concerning the optical properties, the dielectric function shows a sharp increase of oscillator strength around $9 \mathrm{eV}$, consistent with the calculated band structure. We hope that this work will stimulate more experiments on this topic. In particular, the results presented here can be directly compared to photoemission experiments as well as optical spectroscopy. An interesting topic would be to investigate the effect of pressure on the quasiparticle spectra and for the different structures. Also, the sequence of structural transitions predicted in [10] can be reproduced experimentally by using high-pressure facilities.

\section{Acknowledgments}

Supercomputer time was provided by CINES (project gem1100) on the IBM SP4. We also acknowledge VR and STINT for financial support.

\section{References}

[1] Crabtree G W, Dresselhaus M S and Buchanan M V 2004 Phys. Today 5739

[2] Grochala W and Edwards P P 2004 Chem. Rev. 1041283

[3] Richardson T J, Slack J L, Armitage R D, Kostecki R, Farangis B and Rubin M D 2001 Appl. Phys. Lett. 783047

[4] Giebels I A M E, Isidorsson J and Griessen R 2004 Phys. Rev. B 69205111

[5] Abdurahman A, Shukla A and Dolg M 2000 J. Chem. Phys. 1124801

[6] Bernath P F, Shayesteh A, Tereszchuk K and Colin R 2002 Science 2971323

[7] Sampath S, Lantzky K M, Benmore C J, Neuefeind J, Siewenie J E, Egelstaff P A and Yarger J L 2003 J. Chem. Phys. 11912499

[8] Wang X F and Andrews L 2005 Inorg. Chem. 44610

[9] Hantsch U, Winkler B and Milman V 2003 Chem. Phys. Lett. 378343

[10] Vajeeston P, Ravindran P, Kjekshus A and Fjellvag H 2004 Appl. Phys. Lett. 8434

[11] Smith G S, Johnson Q C, Smith D K, Cox D E, Snyder R L, Zhou R S and Zalkin A 1988 Solid State Commun. 67491

[12] Hedin L 1965 Phys. Rev. 139 A796

[13] Hedin L and Lundquist S 1969 Solid State Physics vol 23, ed H Ehrenreich, F Seitz and D Turnbull (New York: Academic) p 1

[14] Lebègue S, Arnaud B, Alouani M and Bloechl P E 2003 Phys. Rev. B 67155208

[15] Arnaud B and Alouani M 2000 Phys. Rev. B 624464

[16] Adler S D 1962 Phys. Rev. 126413

[17] Wiser N 1963 Phys. Rev. 12962

[18] Johnson D L 1974 Phys. Rev. B 94475

[19] Jepsen O and Andersen O K 1971 Solid State Commun. 91763

[20] Lehmann G and Taut M 1972 Phys. Status Solidi b 54469

[21] Arnaud B and Alouani M 2001 Phys. Rev. B 6385208

[22] Aryasetiawan F and Gunnarsson O 1998 Rep. Prog. Phys. 61237

[23] Aulbur W G, Jönsson L and Wilkins J W 1999 Quasiparticle calculations in solids Solid State Phys. 541

[24] Onida G, Reining L and Rubio A 2002 Rev. Mod. Phys. 74601

[25] Lebègue S, Alouani M, Arnaud B and Pickett W E 2003 Europhys. Lett. 63562

[26] Araújo C M, Lebègue S, Eriksson O, Arnaud B, Alouani M and Ahuja R 2005 J. Appl. Phys. 98096106

[27] Blöchl P E 1994 Phys. Rev. B 5017953

[28] Perdew J P, Burke K and Ernzerhof M 1996 Phys. Rev. Lett. 773865

[29] Lebègue S, Arnaud B, Rabiller P, Alouani M and Pickett W E 2004 Europhys. Lett. 68846

[30] Shirley E L 1998 Phys. Rev. B 589579 